Nonlinear Phenomena in Complex Systems, vol. 24, no. 2 (2021), pp. 104 - 111

\title{
Optical Vortices Generation by Azopolymeric Relief Gratings
}

\author{
E. A. Melnikova, ${ }^{*}$ D. V. Gorbach, I. I. Rushnova, V. S. Kabanova, S. S. Slusarenko, and A. L. Tolstik \\ Faculty of Physics, Belarusian State University, \\ 4 Nezalezhnasti Ave., 220030 Minsk, BELARUS \\ C. Losmanschii, A. Meshalkin, and E. Achimova \\ Institute of Applied Physics, 5 Academiei Str, Chisinau MD-2028, MOLDOVA
}

(Received 28 January, 2021)

\begin{abstract}
The energy and spectral conditions for single-stage holographic recording of a diffraction optical element based on the carbazole-containing azo polymer, that forms singular light beams (optical vortices), have been established. With the atomic-force microscopy (AFM), the surface morphology of the recorded relief holograms was studying, and their diffraction efficiency has been estimated. The topology of the generated optical phase singularities has been studied and the stability range of an optical vortex having the topological charge $1=$ 2 has been found. The possibility of using the developed diffractive optical element in the scheme of optical tweezers for manipulating micro-objects is demonstrated.
\end{abstract}

PACS numbers: 42.40.Eq, 42.70.Ln

Keywords: azo polymer, optical vortices, surface relief grating, holographic recording

DOI: https://doi.org/10.33581/1561-4085-2021-24-2-104-111

\section{Introduction}

For the last two decades singular light beams (optical vortices) have attracted considerable interest of scientists and engineers. The studies embrace the generation methods of optical vortices [1-10] and their applications in different scientific and technological applications [1124]. Singular light beams are successfully used to solve the problems associated with optical microparticle manipulations [11-13], data transfer and processing [14-17], laser treatment of materials [18-20], high-resolution microscopy [21, 22], astronomy [23, 24], etc.

One of the techniques to form vortex light beams is the use of diffraction optical elements (DOEs) with a complex amplitude and (or) phase profile. DOEs may be produced as a result of solving the inverse diffraction problem (computer-generated holograms) [1-3] or by means of interference-holographic methods

\footnotetext{
${ }^{*}$ E-mail: melnikova@bsu.by
}

(analog recording of a holographic element) [2-4]. To transform the topological charge of a singular light beam, we can use recording of dynamic holograms [7-9], and also the propagation of a Bessel beam in uniaxial crystals [10].

Because of this, of great importance is a search of effective methods and media for the creation of DOE designed to form singular light beams. The photopolymers based on azo dyes in several works are named among the most promising media [25-30]. Azopolymeric materials enable one to realize the effective direct contact optical recording [25] and holographic recording with a high spatial resolution [26]. The use of azo polymers facilitates development of the technologies aimed at the creation of optical micro- and nanostructures for solution of various problems of photonics [27-30].

In this paper consideration is given to conditions of the effective holographic recording of DOEs based on carbazole-containing azo polymer according to the Leith-Upatnieks scheme. DOE is a relief-phase grating with a $\mathrm{B} \ll$ fork» dislocation 
- vortex hologram. As known, diffracted radiation formed by such elements involves optical vortices with the topological charge that is a multiple of the diffraction order [31].

A single-stage recording process of a relief hologram points to great prospects of using the proposed azo polymer in the technologies associated with copying of holographic elements by direct patterning. Simplicity of recording vortex holograms on the material used determines the effectiveness of using DOE for the subsequent formation of an object wave in the process of recording volume vortex holograms with the desired optical-vortex topology [2,3]. The effect of the photo-induced mass transfer manifesting itself in the photopolymeric material used enables a single-stage (without the need for post-exposure treatment) process of analog manufacturing of a relief-phase vortex hologram. Testing of the recorded vortex hologram in a scheme of optical tweezers has revealed the possibility of the effective optical capture and manipulation of microobjects with the use of a singular optical trap.

\section{Materials and methods}

The light-sensitive azo polymer EPC:DO has been used as a polymeric medium in the process of work for vortex hologram recording. The photopolymer is a compound of the carbazolecontaining monomer N-epoxypropyl carbazole (EPC) and of the azo dye Disperse Orange 3 (4(4-nitrophenylazo)aniline) (DO) [32]. Thin layers of the azo polymer (with a thickness on the order of $600 \mathrm{~nm}$ ) were applied to the surface of glass substrates by spin-coating. The layer thickness was estimated using an atomic force microscope from the depth of the cut of the formed film (Figure 1).

As seen from the AFM-image of the sample under study, a thickness of the EPC:DO azopolymeric film formed on the glass substrate was about $600 \mathrm{~nm}$.

To determine the spectral recording

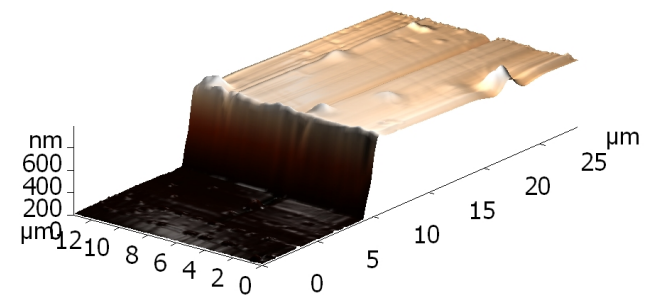

FIG. 1: (color online) AFM image of the cut region of the azopolymer layer.

conditions for DOE, the absorption spectrum of the azopolymeric layer was recorded over the range from $200 \mathrm{~nm}$ to $700 \mathrm{~nm}$ (Figure 2). The data obtained make it possible to find the range of radiation wavelengths for efficient recording of gratings in the region with a high absorption coefficient. On the other hand, the low-absorption region for the azo polymer used determines the spectral range, associated with nondestructive reading of the formed hologram.

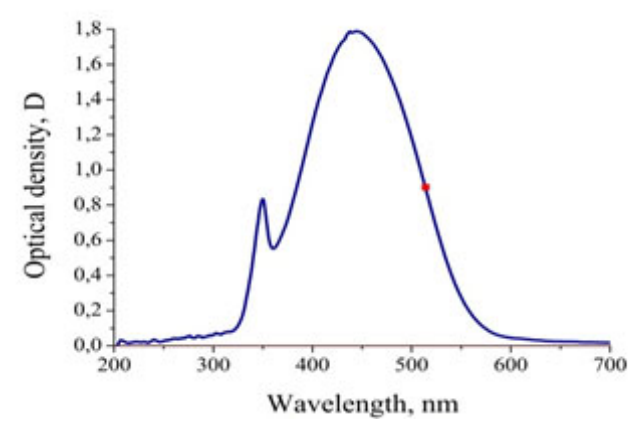

FIG. 2: The absorption spectrum for the film of the EPC:DO azo polymer.

The Nd-YAG laser $(\lambda=532 \mathrm{~nm})$ with the output power radiation of $\mathrm{P}=100 \mathrm{~mW}$ was applied for DOE recording. The holograms were recorded according to the Leith-Upatnieks scheme. The convergence angle was $\theta=5.5^{\circ}$ to provide the condition for recording of a thin phase 
E. A. Melnikova, D. V. Gorbach, I. I. Rushnova, S. S. Slusarenko, A. L. Tolstik, C. Losmanschii,

hologram with the period $\Lambda=6 \mu \mathrm{m}$. To form a fork dislocation, the phase element was placed in one arm of the interferometer to convert the Gaussian intensity distribution of the object beam into an optical vortex with a charge of $l=+1$. On exposure of the azopolymeric film, the light-induced mass transport takes place in the interference region of laser beams, leading to the surface microrelief formation [33, 34]. Optimum conditions for the relief formation on holographic recording are attained with linear polarizations $(p-p)$ of the interfering beams which offer a maximum of the light-induced mass transport in the direction of the interference pattern vector [35]. Orientations of the polarization vector of reference and object waves were controlled by phase half-wave plates. The total power of the interfering light beams was $80 \mathrm{~mW}$ at the interference pattern area of $S=0.04 \mathrm{~cm}^{2}$. Hologram recording was controlled through kinetics of the diffraction efficiency in the process of recording. The monitoring was realized by radiation of a laser diode $(\lambda=650 \mathrm{~nm})$ in which the optical erasure of the relief did not occur.

\section{Results and discussion}

Figure 3 presents a pattern of radiation diffraction from the recorded DOE and the intensity distribution over the cross-sections of diffracted beams in the direction of $K$-th orders $(K=0, \pm 1, \pm 2)$.

Figure 4 shows kinetics of the diffraction efficiency on recording of a relief grating in the EPC:DO azo polymer film and the results obtained during studies of the grating surface morphology based on the AFM method. As seen in Figure 4, during a period of 400 seconds a diffraction efficiency of the formed grating comes to the stationary level. In this case the first-order diffraction efficiency was $\eta_{ \pm}=16 \%$, whereas a depth of the formed relief was $200 \mathrm{~nm}$.

An experimental study of the phase topology of singular light beams formed by the manufactured DOE was carried out according to the Mach-Zehnder interferometer scheme (Figure 5).

To form a singular light beam, the manufactured DOE (Figure 5, pos.7) was positioned at one of the interferometer arms. When recording of the interference pattern of a vortex with a spherical wave was required, collecting lens (Figure 5, pos.4) was positioned at the second interference arm. The intensity distribution was recorded by means of CCDcamera (Figure 5, pos.9).

Figure 6 presents images for the transverse intensity distribution of a light field in the direction of the diffraction order $K=-1$ (left) and the pattern of its interference with a plane (middle) and a spherical (right) wave. It is seen that in the direction of the -1-order diffraction an optical vortex is formed with the topological charge $l_{1}=-1$.

Figure 7 demonstrates images for the transverse intensity distributions of light-field in the direction of the second-order diffraction (a,d), result of their coherent summation with a plane (b, e) and with a spherical (c, f) wave at different distances from DOE.

Based on analysis of the interferograms shown in Figure 7, the second-order diffraction is associated with generation of an optical vortex with the topological charge $l=-2$. To analyze the topological stability of optical vortices, the corresponding interferograms were recorded at different distances from the place of their formation. It has been found that the secondorder optical vortex was instable, breaking into two first-order optical vortices. Note that such a behavior is typical for optical vortices of high orders.

The manufactured DOE was tested using the optical tweezers scheme enabling microparticle manipulations. Aspects of optical capture and singular beam manipulations involve the problems associated with damaging of the captured biomaterial at the specific powers of optical radiation [12]. Destruction of living microobjects is an extremely unwanted effect in biological studies conducted when using 

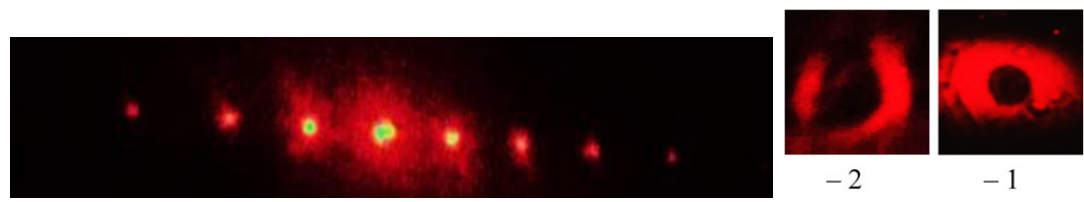

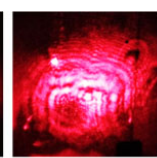

0

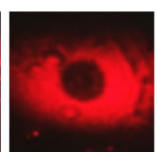

$+1$

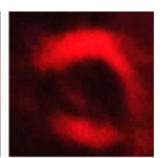

$+2$

FIG. 3: (color online) Radiation diffraction pattern on the recorded DOE (left) and the intensity distribution over the cross-sections of the beams diffracted into different orders $(K=0, \pm 1, \pm 2)$ (right).
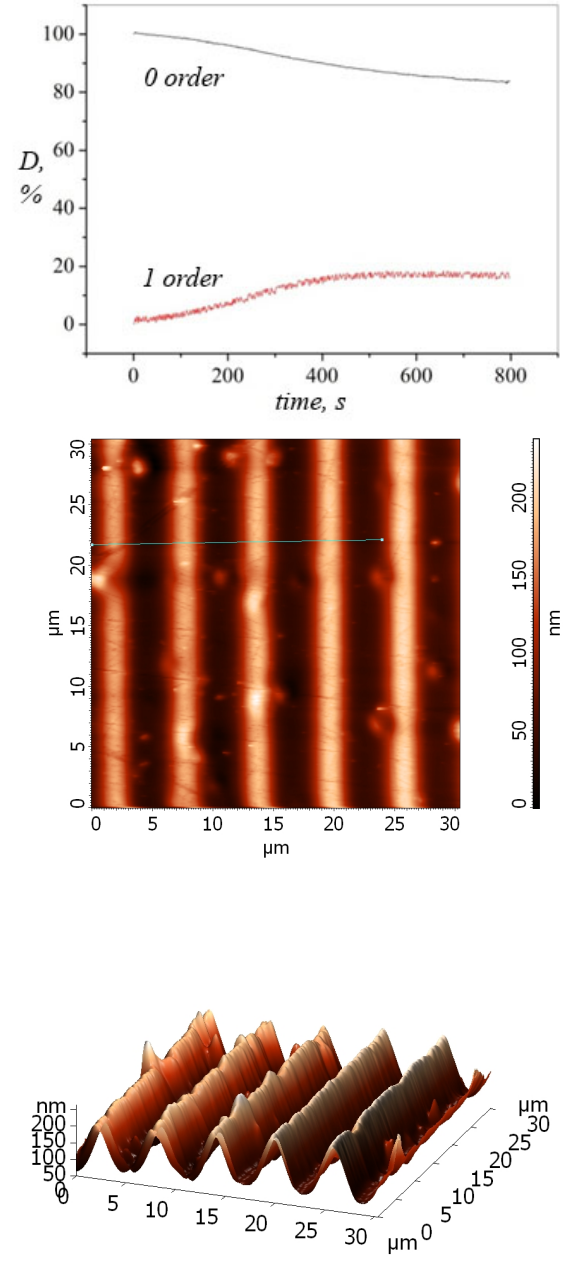

FIG. 4: (color online) Kinetics of the 0-th and the 1-st order diffraction efficiency (up), 3D AFM-image for the relief morphology at a section (down) of hologram (middle).

the optical tweezers scheme. As is know, the beams with the wave-front topology offer more

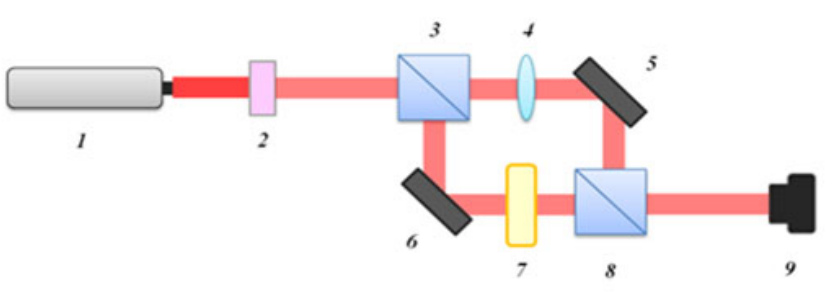

FIG. 5: (color online) Mach-Zehnder interferometer scheme for experimental studies of the phase topology of singular light beams. 1 - He-Ne-laser $(\lambda=632.8 \mathrm{~nm}) ; 2$ - light filter; 3, 8 - beam-splitting cube; 4 - lens; 5, 6 - mirrors; 7 - topological diffraction element on the basis of the azo polymer; 9 - CCD-camera

efficient capturing of microobjects, decreasing the requirements to the power of an optical trap [13].

Figure 8 presents a sequence of images demonstrating capture of microparticles. The object under study was an aqueous solution of yeast cells with the size on the order of $2 \mu \mathrm{m}$. The optical trap was formed by an optical vortex obtained by means of the recorded DOE with a unit topological charge.

Figure 8a shows the optical trap $T$ and localization of yeast cells within the observation plane. The power of the trap-forming beam in the focal plane of the manipulation system was $1 \mathrm{~mW}$, whereas the intensity in the region of the optical trap was about $0.2 \mathrm{MW} / \mathrm{cm}^{2}$. The optical capture process is illustrated by the example of relative microparticle displacements marked in photos as 1 and 2. Horizontal displacement of a box with microobjects in the $X Y$ visualization plane in the direction of decreasing values of the $X$ coordinate has enabled capturing of the cell 
E. A. Melnikova, D. V. Gorbach, I. I. Rushnova, S. S. Slusarenko, A. L. Tolstik, C. Losmanschii, 108 A. Meshalkin, and E. Achimova
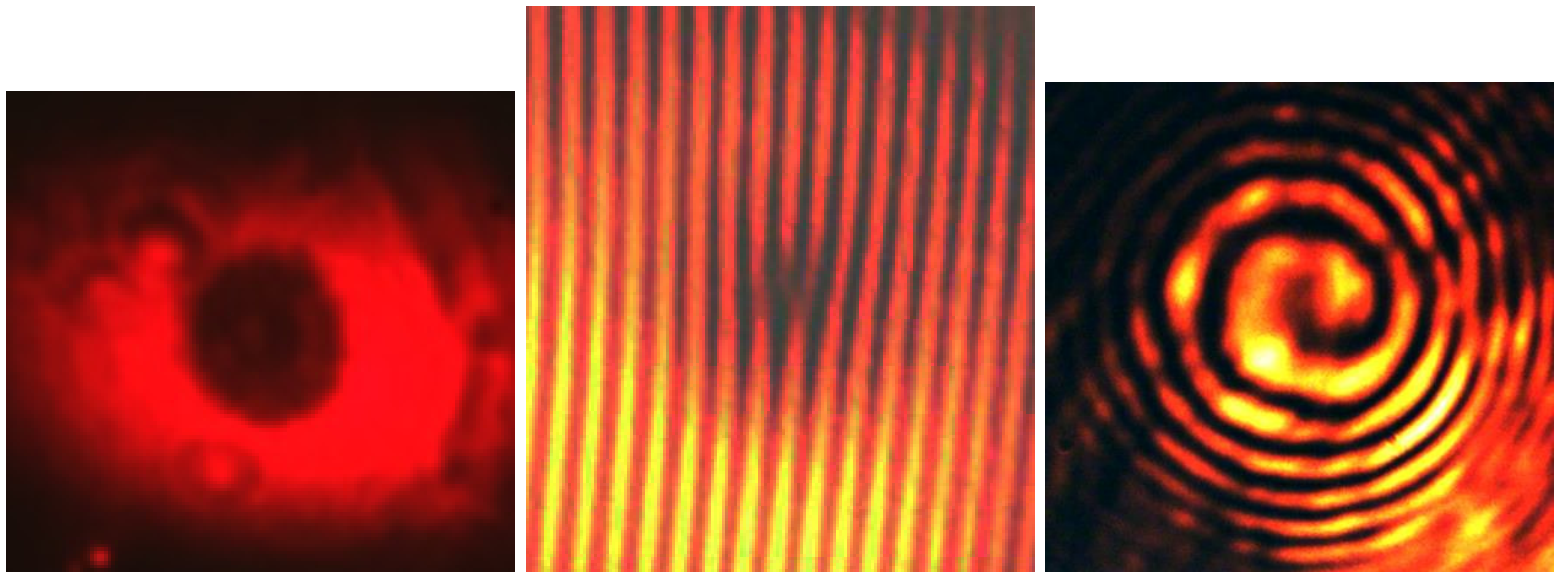

FIG. 6: (color online) Intensity distributions of a light field (left) and patterns of interference of a singular light beam with a plane (middle) and with a spherical wave (right) when using the first-order diffraction.

(a)

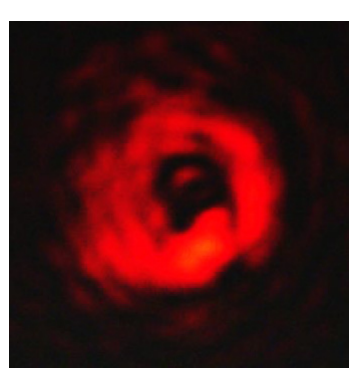

(d)

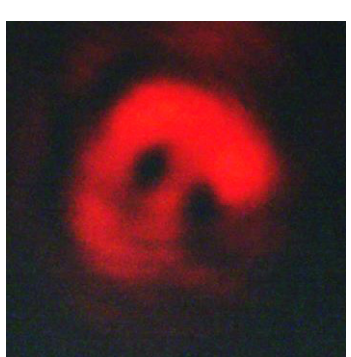

(b)

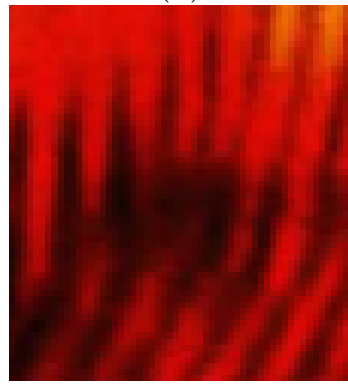

(e)

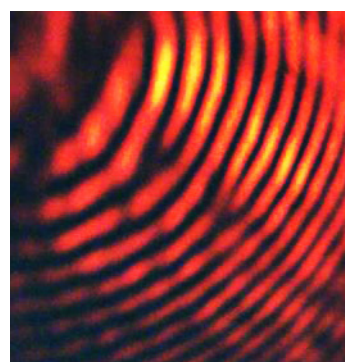

(c)

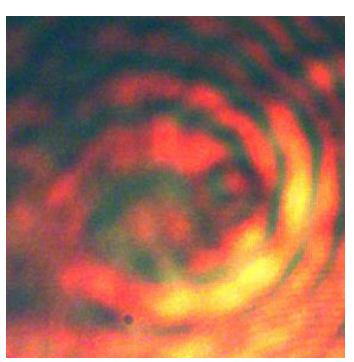

(f)

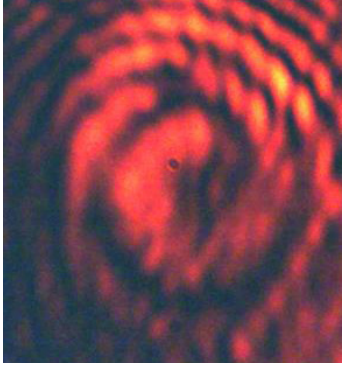

FIG. 7: (color online) Intensity distribution profiles of a light field (a,d) and experimental patterns of interference of a light beam (second-order diffraction) with a plane (b, e) and with a spherical (c, f) wave at a distance of $17 \mathrm{~cm}(\mathrm{a}, \mathrm{b}, \mathrm{c})$ and $22 \mathrm{~cm}(\mathrm{~d}, \mathrm{e}, \mathrm{f})$.

marked as 1 (Fig. 8b). After the capture, the box with microobjects was moving along the vertical axis $Y$ at the rate $1 \mu \mathrm{m} / \mathrm{s}$. As seen from images $8 \mathrm{~b}$ and $8 \mathrm{c}$, the microparticle 1 is rigidly captured by the vortex trap, its position within the $X Y$ plane is invariable as the box is moving, whereas the particle 2 is displaced relative to its initial position. 
(a)

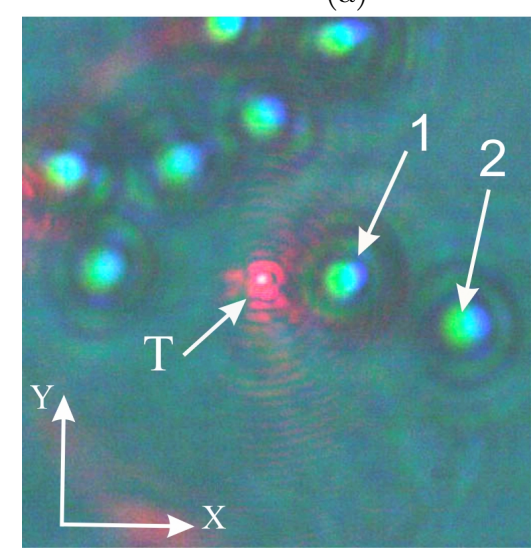

(b)

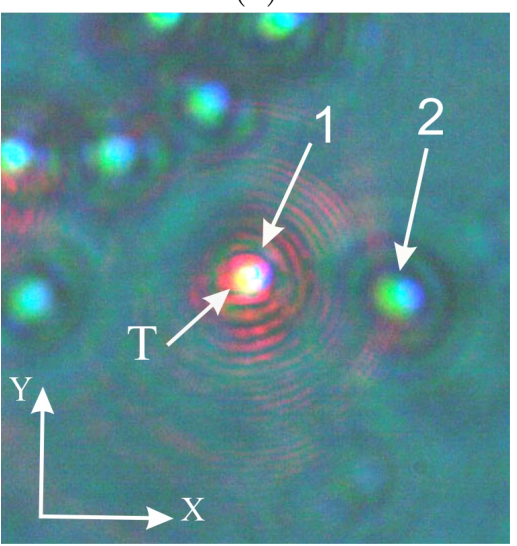

(c)

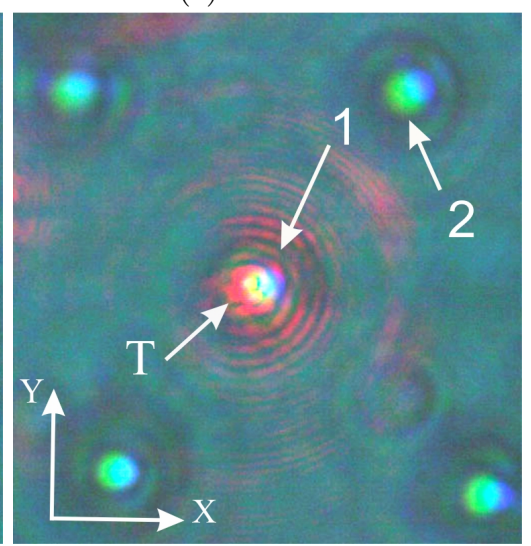

FIG. 8: (color online) Capturing and manipulations with yeast cells by means of the vortex optical trap: a - photos of the optical vortex trap $T$ and of the yeast cells 1 and 2 ; b - capturing of a yeast cell by the vortex optical trap; c - retention of a yeast cell by the vortex optical trap.

\section{Conclusion}

In this way, with the use of the azo polymer EPC:DO as a holographic medium, we can realize the effective single-step recording of DOE that represents a relief-phase vortex hologram. The optical vortices formed on the basis of thin relief holograms make it possible to realize manipulations with biological objects under illumination of light beam with the power of $1 \mathrm{~mW}$. The obtained results may be applied and disseminated in the technologies of copying thin relief holograms or of recording volume vortex holograms, as well as in the technologies of optical microobject manipulations.

\section{Acknowledgment}

The reported study was funded as part of an international bilateral Belarus-Moldova project "Design and development of the "vortex" diffraction optical elements on thin azopolymer layers for biomedicine" F19MLDG-006 and ANCD 1980013.50.07.04A/BL.

\section{References}

[1] R. Heckenberg, R. McDuff, C. Smith, and A. White, Generation of optical phase singularities by computer-generated holograms. Opt. Lett. 17, 221 (1992).

[2] E.A.Melnikova, J. Kurapov, O. Romanov, and A. Toustsik, Holographic and diffractive optical elements for the formation of the vortex and Bessel light beams, Holography. Science and practice, Proceedings of the 11th International conference "HoloExpo'2014" p. 99 (2014).

[3] A.A.Kazak, E.A.Melnikova, O.G.Romanov, and
A.L.Tolstik, Formation of singular and Bessel light beams using electrically controlled liquid crystal diffractive elements. Int. J. Nonlinear Phenomena in Complex Systems 18, 170 (2015).

[4] M. D. Levenson, T. Ebihara, G. Dai, Y. Morikawa, N. Hayashi, and S. M. Tan, Optical vortex masks for via levels. J. Microlith., Microfab., Microsys. 3(2), 293 (2004).

[5] Z. Shen, R. Li, Y. Z. Xue, Z. Y. Qiu, Z. Y. Xiang, J. Y. Zhou, and B. F. Zhang, Generation of Optical Vortices With Polarization-Insensitive 
E. A. Melnikova, D. V. Gorbach, I. I. Rushnova, S. S. Slusarenko, A. L. Tolstik, C. Losmanschii, 110 A. Meshalkin, and E. Achimova

Metasurfaces, IEEE Photonics J. 12, 1 (2020).

[6] D. Lee, H. Lee, L. K. Migara, K. Kwak, V. P. Panov, and J. Song, Widely Tunable Optical Vortex Array Generator Based on Grid Patterned Liquid Crystal Cell, Adv. Opt. Mater. p. 2001604 (2020).

[7] D.V.Gorbach, S.A.Nazarov, O.G.Romanov, and A.L.Tolstik, Polarization transformation of singular light beams upon four- and six-wave mixing. Int. J. Nonlinear Phenomena in Complex Systems 18, 149 (2015).

[8] O.G.Romanov, D.V.Gorbach, and A.L.Tolstik, Transformation of optical vortices by polarization dynamic holograms. Optics and Spectroscopy 115, 335 (2013).

[9] A.L.Tolstik, Singular Dynamic Holography. Russian Physics Journal 58, 1431 (2016).

[10] S. Nazarov, D. Gorbach, E. Mel'nikova, S. Kurilkina, and A. Tolstik, Spin-orbit transformation of Bessel light beams by electrically controlled liquid-crystal elements. Journal of Optical Technology 85 (4), 189 (2018).

[11] D. G. Grier, A revolution in optical manipulation. Nature 424, 810 (2003).

[12] G. Leitz, E. Fallman, S. Tuck, and O. Axner, Stress response in Caenorhabditis elegans caused by optical tweezers: wavelength, power, and time dependence. Biophys. J. 82, 2224 (2002).

[13] Y. Jiang, K. Huang, and X. Lu, Radiation force of highly focused Lorentz-Gauss beams on a Rayleigh particle. Opt. Express 19, 9708 (2011).

[14] J. Wang, Terabit free-space data transmission employing orbital angular momentum multiplexing. Nat, Photonics 6, 488 (2012).

[15] N. Bozinovic, Terabit-scale orbital angular momentum mode division multiplexing in fibers. Science 340, 1545 (2013).

[16] G. Molina-Terriza, J. P. Torres, and L. Torner, Twisted photons, Nat. Phys. 3, 305 (2007).

[17] M. Erhard, Twisted photons: new quantum perspectives in high dimensions. Light Sci. Appl. 7, 17146 (2018).

[18] F. Takahashi, Picosecond optical vortex pulse illumination forms a monocrystalline silicon needle. Sci. Rep. 6, 21738 (2016).

[19] K. Toyoda, Transfer of light helicity to nanostructures. Phys. Rev. Lett. 110, 143603 (2013).

[20] L. Yang, Direct laser writing of complex microtubes using femtosecond vortex beams. Appl. Phys. Lett. 110, 221103 (2017).
[21] F. Tamburini, Overcoming the Rayleigh criterion limit with optical vortices. Phys. Rev. Lett. 97, 163903 (2006).

[22] K. I. Willig, STED microscopy reveals that synaptotagmin remains clustered after synaptic vesicle exocytosis. Nature 440, 935 (2006).

[23] G.Foo, D.M.Palacios, and G. Swartzlander, Optical vortex coronagraph. Opt. Lett. 30, 3308 (2005).

[24] F. Tamburini, Twisting of light around rotating black holes. Nat. Phys. 7, 195 (2011).

[25] O. Kabanova, I. Rushnova, E. Melnikova, A. Tolstik, A. Muravsky, A. Murauski, and R.Heintzmann, Two-Dimentional Diffractive Optical Structure Based on Patterned Photoalignment of Polymerizable Liquid Crystal, J. Belarusian State Univ. Phys. 3, 4 (2019).

[26] A. Sobolewska, S. Bartkiewicz, and A. Priimagi, High-Modulation-Depth Surface Relief Gratings Using s - s Polarization Configuration in Supramolecular Polymer-Azobenzene Complexes, J. Phys. Chem. 118, 23279 (2014).

[27] A. Priimagi and A. Shevchenko, AzopolymerBased Micro- and Nanopatterning for Photonic Applications, J. Polym. Sci. Part B Polym. Phys. 52, 163 (2014).

[28] V. Pagliarulo, A. Calabuig, S. Grilli, and P. Ferraro, Direct Quantitative Imaging of the Writing Stage in a Photosensitive Azopolymer by Digital Holography, Soft Matter 15, 7809 (2019).

[29] M. Salvatore, F. Borbone, and S. L. Oscurato, Deterministic Realization of Quasicrystal Surface Relief Gratings on Thin Azopolymer Films, Adv. Mater. Interfaces 7, 1902118 (2020).

[30] K. Kim, Y. M. Lee, M. Ryou, and Y. Jeong, Direct Fabrication of Nanodomes-Combined Surface Relief Gratings on Azobenzene Polymer Films with Controlled Shapes and Sizes, J. Polym. Sci. Part B Polym. Phys. 57, 731 (2019).

[31] V. Bazhenov, M.V.Vasnetsov, and M. Soskin, Laser beams with screw dislocations in their wavefronts, JETP Letters 52, 429 (1990).

[32] A. Meshalkin, S. Robu, E. Achimova, A. Prisacar, D. Shepel, V. Abaskin, and G. Triduh, Direct Photoinduced Surface Relief Formation in Carbazole-Based Azopolymer Using Polarization Holographic Recording, J. Optoelectron. Adv. Mater. 18, 763 (2016).

[33] N. Viswanathan, D. Kim, S. Bian, J. Williams, W. Liu, L. Li, L. Samuelson, J. Kumar, and S. Tripathy, Surface Relief Structures on Azo 
Polymer Films, J. Mater. Chem. 9, 1941 (1999).

[34] A. Sobolewska, S. Bartkiewicz, A. Miniewicz, and E. Schab-Balcerzak, Polarization Dependence of Holographic Grating Recording in Azobenzene-Functionalized Polymers Monitored by Visible and Infrared Light, J. Phys. Chem. B
114, 9751 (2010).

[35] V. Kryshenik, Y. Azhniuk, and V. Kovtunenko, All-Optical Patterning in Azobenzene Polymers and Amorphous Chalcogenides. J. Non. Cryst. Solids 512, 112 (2019). 